\title{
Measures of entanglement in multipartite bound entangled states
}

\author{
Tzu-Chieh Wei, ${ }^{1}$ Joseph B. Altepeter, ${ }^{1}$ Paul M. Goldbart, ${ }^{1}$ and William J. Munro ${ }^{2}$ \\ ${ }^{1}$ Department of Physics, University of Illinois at Urbana-Champaign, \\ 1110 West Green Street, Urbana, Illinois 61801-3080, USA \\ ${ }^{2}$ Hewlett-Packard Laboratories, Filton Road, Stoke Gifford, Bristol, BS34 SQ2, United Kingdom
}

(Dated: November 5, 2018)

\begin{abstract}
Bound entangled states are states that are entangled but from which no entanglement can be distilled if all parties are allowed only local operations and classical communication. However, in creating these states one needs nonzero entanglement resources to start with. Here, the entanglement of two distinct multipartite bound entangled states is determined analytically in terms of a geometric measure of entanglement and a related quantity. The results are compared with those for the negativity and the relative entropy of entanglement.
\end{abstract}

PACS numbers: 03.67.Mn, 03.65.Ud

Introduction - We are motivated to study the quantification of entanglement for the basic reason that entanglement has been identified as a resource central to much of quantum information processing (see, e.g., Ref. [1]). To date, progress in the quantification of entanglement for mixed states has resided primarily in the domain of bipartite systems [2]. For multipartite systems in pure and mixed states, the characterization and quantification of entanglement present even greater challenges.

Among all entangled states, there is a peculiar class of states, bound entangled states, originally discovered in the bipartite setting, where some connection to zero negativity was identified [3]. These are states that are entangled, but from which no pure entangled state can be distilled if all parties are allowed only local operations and classical communication (LOCC). The distillable entanglement $\left(E_{\mathrm{D}}\right)$ is thus zero. Bound entangled states can be either bipartite or multipartite, the latter possibly exhibiting more structure than the former. However, it does take nonzero entanglement to create bound entangled states under LOCC. It is thus desirable to see how much entanglement is needed.

However, the two most important measuresentanglement of distillation $\left(E_{\mathrm{D}}\right)$ and of formation $\left(E_{\mathrm{F}}\right)$ - have so far been limited to bipartite settings, as there are ambiguities in generalizing them to multipartite systems 4]. In order to explore multipartite settings, it is thus, on the one hand, necessary to lay down bounds on the entanglement content for distillation and formation. On the other hand, applying other measures, such as the relative entropy of entanglement $\left(E_{\mathrm{R}}\right)$, to multipartite states may prove helpful in quantifying entanglement. Recently, a multipartite entanglement measure based on the geometry of Hilbert space has been proposed [5, 6, 7], and has been applied to several bipartite and multipartite cases. The merit of this measure is that it is suitable for any-partite systems with any dimension, although determining it analytically for generic states remains a challenge.

In the present paper, we study the entanglement con- tent of two distinct bound entangled states: Smolin's four-party unlockable bound entangled state [8, 9] and Dür's $N$-party Bell-inequality-violating bound entangled states [10]. For each, we determine analytically their geometric measure of entanglement and a related quantity. Under certain circumstances, these give lower bounds on their multipartite $E_{\mathrm{F}}$. In addition, we make conjectures concerning their relative entropies of entanglement. Although quantities such as the geometric measure or the relative entropy of entanglement may not be able to reveal the exact nature of bound entanglement, they nevertheless quantify for these bound entangled states the content of entanglement that is unextractable.

Geometric measure of entanglement - We begin by briefly reviewing its formulation. Consider a general $n$-partite pure state (expanded in the local bases $\left\{e_{p_{i}}^{(i)}\right\}$ ):

$$
|\psi\rangle=\sum_{p_{1} \cdots p_{n}} \chi_{p_{1} p_{2} \cdots p_{n}}\left|e_{p_{1}}^{(1)} e_{p_{2}}^{(2)} \cdots e_{p_{n}}^{(n)}\right\rangle
$$

As shown in Ref. 7], its closest separable (i.e., product) pure state (with $i$ being the party index)

$$
|\phi\rangle \equiv \underset{i=1}{\otimes}\left|\phi^{(i)}\right\rangle=\bigotimes_{i=1}^{n}\left(\sum_{p_{i}} c_{p_{i}}^{(i)}\left|e_{p_{i}}^{(i)}\right\rangle\right)
$$

satisfies the condition (and its complex conjugate)

$$
\sum_{p_{1} \cdots \widehat{p}_{i} \cdots p_{n}} \chi_{p_{1} p_{2} \cdots p_{n}}^{*} c_{p_{1}}^{(1)} \cdots \widehat{c_{p_{i}}^{(i)}} \cdots c_{p_{n}}^{(n)}=\Lambda c_{p_{i}}^{(i)^{*}}
$$

where the eigenvalue $\Lambda \in[-1,1]$ is associated with the Lagrange multiplier enforcing $\langle\phi \mid \phi\rangle=1$, and the caret denotes exclusion. Moreover, $\Lambda$ is the cosine of the angle between $|\psi\rangle$ and $|\phi\rangle$; the largest one $\Lambda_{\max }$, which we call the entanglement eigenvalue, corresponds to the closest separable state, and is the maximal overlap: $\Lambda_{\max }(|\psi\rangle)=\max _{\phi}|\langle\phi \mid \psi\rangle|$, where $|\phi\rangle$ is separable but otherwise arbitrary. $E_{\sin ^{2}} \equiv 1-\Lambda_{\max }^{2}(|\psi\rangle)$ was defined to be the geometric measure of entanglement [7] for state $|\psi\rangle$, and it measures the degree of inseparability 
via the squared sine of the angle away from the closest separable pure state.

The extension to mixed states can be built upon the pure-state theory and is made via the use of the convex hull construction, as was done for $E_{\mathrm{F}}$ [2]. The essence is a minimization over all decompositions $\rho=\sum_{i} p_{i}\left|\psi_{i}\right\rangle\left\langle\psi_{i}\right|$ into pure states:

$$
E_{\sin ^{2}}(\rho) \equiv \min _{\left\{p_{i}, \psi_{i}\right\}} \sum_{i} p_{i} E_{\sin ^{2}}\left(\left|\psi_{i}\right\rangle\right)
$$

This convex hull construction ensures that the measure gives zero for unentangled states; however, it also complicates the task of determining mixed-state entanglement. $E_{\sin ^{2}}$ was shown to be an entanglement monotone [6, 7] (i.e., the measure does not increase under LOCC), hence is a good measure of entanglement. As there is no explicit generalization of $E_{\mathrm{F}}$ to multipartite states, we shall calculate $E_{\sin ^{2}}$ analytically for two bound entangled states, Smolin's and Dür's. Because $E_{\mathrm{F}}(\rho)$ is the minimum average ebit to create a single copy of $\rho$, we can regard $E_{\sin ^{2}}(\rho)$ as the minimum average degree of pure inseparability needed to realize the state $\rho$.

In bipartite settings, it is known [1] that $E_{\mathrm{R}}(\rho) \leq$ $E_{\mathrm{F}}(\rho)$, and that for pure states $|\psi\rangle, E_{\mathrm{R}}(\psi)=E_{\mathrm{F}}(\psi)$. It is also known 12 that for any bi- and multipartite pure state $|\psi\rangle, \mathcal{E}_{\log _{2}}(\psi) \equiv-2 \log _{2} \Lambda_{\max }(\psi) \leq E_{\mathrm{R}}(\psi)$. Together with the inequality $\left(1-x^{2}\right) \log _{2} e \leq-2 \log _{2} x$ (for $0 \leq x \leq 1$ ), one has

$$
\sum_{i} p_{i} E_{\sin ^{2}}\left(\psi_{i}\right) \log _{2} e \leq \sum_{i} p_{i} \mathcal{E}_{\log _{2}}\left(\psi_{i}\right) \leq \sum_{i} p_{i} E_{\mathrm{F}}\left(\psi_{i}\right),
$$

and thus $\left(\log _{2} e\right) E_{\sin ^{2}}(\rho) \leq \mathcal{E}_{\log _{2}}(\rho) \leq E_{\mathrm{F}}(\rho)$ for any $b i$ partite state $\rho$. If the generalization of $E_{\mathrm{F}}$ to multipartite states maintains the property that $E_{\mathrm{F}}(\psi) \geq E_{\mathrm{R}}(\psi)$ then the inequality $\left(\log _{2} e\right) E_{\sin ^{2}} \leq \mathcal{E}_{\log _{2}} \leq E_{\mathrm{F}}$ will continue to hold for multipartite mixed states. We remark that

$$
\mathcal{E}_{\log _{2}}(\rho) \equiv \min _{\left\{p_{i}, \psi_{i}\right\}} \sum_{i} p_{i}\left(-2 \log _{2} \Lambda_{\max }\left(\psi_{i}\right)\right)
$$

is not an entanglement monotone [12]. However, we see that both $\left(\log _{2} e\right) E_{\sin ^{2}}$ and $\mathcal{E}_{\log _{2}}$ could serve as lower bounds on multipartite entanglement of formation.

We now turn to the calculations of entanglement for the two bound entangled states: Smolin's and Dür's.

Smolin's four-party unlockable bound entangled stateThis is a four-qubit mixed state

$$
\rho^{A B C D} \equiv \frac{1}{4} \sum_{i=0}^{3}\left(\left|\Psi_{i}\right\rangle\left\langle\Psi_{i}\right|\right)_{\mathrm{AB}} \otimes\left(\left|\Psi_{i}\right\rangle\left\langle\Psi_{i}\right|\right)_{\mathrm{CD}}
$$

where the $|\Psi\rangle$ 's are the four Bell states $(|00\rangle \pm|11\rangle) / \sqrt{2}$ and $(|01\rangle \pm|10\rangle) / \sqrt{2}$. Now, the state $\rho^{A B C D}$ can be conveniently rewritten as

$$
\rho^{A B C D}=\frac{1}{4} \sum_{i=0}^{3}\left|X_{i}\right\rangle\left\langle X_{i}\right|,
$$

where the $|X\rangle$ 's are the four orthogonal GreenbergerHorne-Zeilinger-(GHZ) like states:

$$
\begin{aligned}
& \left|X_{0}\right\rangle \equiv \frac{1}{\sqrt{2}}(|0000\rangle+|1111\rangle),\left|X_{1}\right\rangle \equiv \frac{1}{\sqrt{2}}(|0011\rangle+|1100\rangle), \\
& \left|X_{2}\right\rangle \equiv \frac{1}{\sqrt{2}}(|0101\rangle+|1010\rangle),\left|X_{3}\right\rangle \equiv \frac{1}{\sqrt{2}}(|0110\rangle+|1001\rangle) .
\end{aligned}
$$

The most general decomposition of a mixed state $\rho$ into pure states can be expressed as

$$
\rho=\sum_{k=1}^{\mathcal{M}}\left|\tilde{\varphi}_{k}\right\rangle\left\langle\tilde{\varphi}_{k}\right| \text { with }\left|\tilde{\varphi}_{k}\right\rangle=\sum_{i=1}^{n} \mathcal{U}_{k i} \sqrt{\lambda_{i}}\left|\xi_{i}\right\rangle,
$$

where $\mathcal{M}$ is an integer not smaller than $n$, the number of orthonormal eigenvectors $\left\{\left|\xi_{i}\right\rangle\right\}$ (with nonzero eigenvalues $\left.\left\{\lambda_{i}\right\}\right)$ of $\rho$, the $|\tilde{\varphi}\rangle$ 's are unnormalized, and $\mathcal{U}$ satisfies $\sum_{k=1}^{\mathcal{M}} \mathcal{U}_{k i} \mathcal{U}_{k j}^{*}=\delta_{i j}$. Thus, the most general pure state that appears in the decomposition of Smolin's state is

$$
\left|\tilde{\varphi}_{k}\right\rangle=\sum_{i=0}^{3} \frac{1}{2} \mathcal{U}_{k i}\left|X_{i}\right\rangle
$$

Our goal is to minimize $\sum_{k} p_{k} E_{\text {pure }}\left(\left|\varphi_{k}\right\rangle\right)$ over all possible $\mathcal{U}$ 's, where $E_{\text {pure }}$ is some pure-state entanglement $\left(E_{\sin ^{2}}\right.$ or $\mathcal{E}_{\log _{2}}$ in our considerations), $p_{k} \equiv\left\langle\tilde{\varphi}_{k} \mid \tilde{\varphi}_{k}\right\rangle$, and $\left|\varphi_{k}\right\rangle$ is the normalized state $\left|\varphi_{k}\right\rangle \equiv\left|\tilde{\varphi}_{k}\right\rangle / \sqrt{p_{k}}$. Making a general minimization for an arbitrary mixed state is extremely difficult. However, for the mixed state $\rho^{A B C D}$ we shall show that the decomposition in Eq. (7) does indeed minimize the average entanglement over pure-state decompositions. As in Eq. (9), $|\varphi\rangle$ can be explicitly written as $|\varphi\rangle=\sum_{i=0}^{3} \sqrt{q_{i}} e^{i \phi_{i}}\left|X_{i}\right\rangle$, where the $q$ 's are nonnegative, satisfying $\sum_{i} q_{i}=1$, and the $\phi$ 's are phases. For fixed $q$ 's, the state has a maximal entanglement eigenvalue when all phases are zero. We shall show shortly that its maximal entanglement eigenvalue is $1 / \sqrt{2}$, which is achieved by the $|X\rangle$ 's.

The entanglement eigenvalue of the state $|\varphi\rangle=$ $\sum_{i=0}^{3} \sqrt{q_{i}}\left|X_{i}\right\rangle$ is the maximal overlap with the separable state $|\Phi\rangle=\otimes_{i=1}^{4}\left(c_{i}|0\rangle+s_{i}|1\rangle\right)$, where $c_{i} \equiv \cos \theta_{i}$ and $s_{i} \equiv \sin \theta_{i}$ with $0 \leq \theta_{i} \leq \pi / 2$. Thus

$$
\begin{aligned}
\langle\Phi \mid \varphi\rangle= & \sqrt{q_{0} / 2}\left(c_{1} c_{2} c_{3} c_{4}+s_{1} s_{2} s_{3} s_{4}\right)+\sqrt{q_{1} / 2}\left(c_{1} c_{2} s_{3} s_{4}\right. \\
& \left.+s_{1} s_{2} c_{3} c_{4}\right)+\sqrt{q_{2} / 2}\left(c_{1} s_{2} c_{3} s_{4}+s_{1} c_{2} s_{3} c_{4}\right) \\
& +\sqrt{q_{3} / 2}\left(c_{1} s_{2} s_{3} c_{4}+s_{1} c_{2} c_{3} s_{4}\right),
\end{aligned}
$$

which has maximum $1 / \sqrt{2}$. To see this, use the Cauchy-Schwarz inequality, treating as one vector $\left\{\sqrt{q_{0} / 2}, \sqrt{q_{1} / 2}, \sqrt{q_{2} / 2}, \sqrt{q_{3} / 2}\right\}$ (whose modulus is $1 / \sqrt{2}$ ), and the corresponding coefficients as another vector (whose modulus can be shown to be no greater than 1; see App. A). The states $\left|X_{i}\right\rangle$ clearly saturate this bound; hence:

$$
E_{\sin ^{2}}\left(\rho^{A B C D}\right)=1 / 2, \quad \mathcal{E}_{\log _{2}}\left(\rho^{A B C D}\right)=1 .
$$


This suggests that although bound entangled, Smolin's state has a very high degree of entanglement, the same as that of a 4-partite GHZ state. This high degree of entanglement seems to manifest in some bipartite partitioning, e.g., $\{\mathrm{A}: \mathrm{BCD}\}$ (as we discuss below).

We conjecture (and later prove) that $E_{\mathrm{R}}=1$ for this state and one of its closest separable mixed states is

$\frac{1}{8}(|0000\rangle\langle 0000|+| 1111\rangle\langle 1111|+| 0011\rangle\langle 0011|+| 1100\rangle\langle 1100|$

$+|0101\rangle\langle 0101|+| 1010\rangle\langle 1010|+| 0110\rangle\langle 0110|+| 1001\rangle(1001 \mid)$.

We remark that the negativity $\mathcal{N}$ [2] (a value used to quantify the degree of bipartite inseparability of states and defined as twice the absolute sum of negative eigenvalues of the partial transpose (PT) of the density matrix with respect to some bipartite partitioning) is zero for any $2 / 2$ partitioning, e.g., $\{\mathrm{AB}: \mathrm{CD}\}$, but nonzero for $1 / 3$ partitioning, e.g., $\{\mathrm{A}: \mathrm{BCD}\}$. Specifically, $\mathcal{N}_{\mathrm{A}: \mathrm{BCD}}=$ 1 but $\mathcal{N}_{\mathrm{AB}: \mathrm{CD}}=0$.

Let us now turn to Dür's bound entangled states.

Dür's N-party bound entangled states - Dür [10] found that for $N \geq 4$ the following state is bound entangled:

$$
\rho_{N} \equiv \frac{1}{N+1}\left(\left|\Psi_{G}\right\rangle\left\langle\Psi_{G}\right|+\frac{1}{2} \sum_{k=1}^{N}\left(P_{k}+\bar{P}_{k}\right)\right),
$$

where $\left|\Psi_{G}\right\rangle \equiv\left(\left|0^{\otimes N}\right\rangle+e^{i \alpha_{N}}\left|1^{\otimes N}\right\rangle\right) / \sqrt{2}$ is a $N$-partite GHZ state; $P_{k} \equiv\left|u_{k}\right\rangle\left\langle u_{k}\right|$ is a projector onto the state $\left|u_{k}\right\rangle \equiv|0\rangle_{1}|0\rangle_{2} \ldots|1\rangle_{k} \ldots|0\rangle_{N}$; and $\bar{P}_{k} \equiv\left|v_{k}\right\rangle\left\langle v_{k}\right|$ projects on to $\left|v_{k}\right\rangle \equiv|1\rangle_{1}|1\rangle_{2} \ldots|0\rangle_{k} \ldots|1\rangle_{N}$. For $N \geq$ 8 this state violates the Mermin-Klyshko-Bell inequality [10]; violation was pushed down to $N \geq 7$ by Kaszlikowski et al. 13] for a three-setting Bell inequality; it was pushed further down to $N \geq 6$ by Sen et al. 14] for a functional Bell inequality. The phase $\alpha_{N}$ in $\left|\Psi_{G}\right\rangle$ can be eliminated by local unitary transformations, and hence we shall take $\alpha_{N}=0$ in the following discussion.

In fact, if we consider the family of states

$$
\rho_{N}(x) \equiv x\left|\Psi_{G}\right\rangle\left\langle\Psi_{G}\right|+\frac{1-x}{2 N} \sum_{k=1}^{N}\left(P_{k}+\bar{P}_{k}\right),
$$

we find that for $N \geq 4$ the state is bound entangled if $0<x \leq 1 /(N+1)$ and is still entangled but not bound entangled if $x>1 /(N+1)$. This can be seen from the fact that the negativities of $\rho_{N}(x)$ with respect to the two different partitions $(1: 2 \cdots N)$ and $(12: 3 \cdots N)$ are

$$
\begin{aligned}
& \mathcal{N}_{1: 2 \cdots N}\left(\rho_{N}(x)\right)=\max \{0,[(N+1) x-1] / N\}, \\
& \mathcal{N}_{12: 3 \cdots N}\left(\rho_{N}(x)\right)=x .
\end{aligned}
$$

By applying arguments similar to those used to calculate entanglement for Smolin's state, we have that the general pure state in the decomposition of $\rho_{N}(x)$ is

$$
\sqrt{y} e^{i \phi_{0}}\left|\Psi_{G}\right\rangle+\sqrt{1-y} \sum_{k=1}^{N}\left(\sqrt{q_{k}} e^{i \phi_{i}}\left|u_{i}\right\rangle+\sqrt{r_{k}} e^{i \phi_{i}^{\prime}}\left|v_{i}\right\rangle\right),
$$

where the $q$ 's and $r$ 's are non-negative and satisfy $\sum_{k}\left(q_{k}+r_{k}\right)=1$. In this family, the state with the least entanglement (or maximum $\Lambda_{\max }$ ) for fixed $\left\{y, q_{k}, r_{k}\right\}$ is the one with all phase factors zero:

$|\Psi(y,\{q, r\})\rangle \equiv \sqrt{y}\left|\Psi_{G}\right\rangle+\sqrt{1-y} \sum_{k=1}^{N}\left(\sqrt{q_{k}}\left|u_{i}\right\rangle+\sqrt{r_{k}}\left|v_{i}\right\rangle\right)$.

Next, we ask: For fixed $y$, what is the least entanglement that the above state can have? Take a separable state of the form $|\Phi\rangle=\otimes_{i=1}^{N}\left(c_{i}|0\rangle+s_{i}|1\rangle\right)$; its overlap with $|\Psi(y,\{q, r\})\rangle$ is then

$$
\begin{aligned}
& \langle\Psi \mid \Phi\rangle=\sqrt{y / 2}\left(c_{1} \cdots c_{N}+s_{1} \cdots s_{N}\right) \\
& +\sqrt{1-y} \sum_{k=1}^{N}\left(\sqrt{q_{k}} c_{1} \cdots s_{k} \cdots c_{N}+\sqrt{r_{k}} s_{1} \cdots c_{k} \cdots s_{N}\right) .
\end{aligned}
$$

This can be shown to be no greater than $\sqrt{(2-y) / 2}$, again by a Cauchy-Schwarz inequality, taking

$$
\left\{\sqrt{y / 2},\left\{\sqrt{(1-y) q_{k}}\right\},\left\{\sqrt{(1-y) r_{k}}\right\}\right\}
$$

as the first $(2 N+1)$-component vector (with modulus $\sqrt{(2-y) / 2}$ and the corresponding coefficients as the second one (whose modulus can be shown to be no greater than 1 for $N \geq 4$; see App. A). The bound can be saturated, e.g., by

$$
\begin{aligned}
\left|\psi_{ \pm, u, k}(y)\right\rangle & \equiv \sqrt{y}\left|\Psi_{G}\right\rangle \pm \sqrt{1-y}\left|u_{k}\right\rangle, \\
\left|\psi_{ \pm, v, k}(y)\right\rangle & \equiv \sqrt{y}\left|\Psi_{G}\right\rangle \pm \sqrt{1-y}\left|v_{k}\right\rangle,
\end{aligned}
$$

for which $\Lambda_{\max }(y)=\sqrt{(2-y) / 2}$ 15. As $1-\Lambda_{\max }^{2}(y)$ is linear in $y$ and $-2 \log _{2} \Lambda_{\max }(y)$ is convex in $y$, one gets

$$
E_{\sin ^{2}}\left(\rho_{N}(x)\right)=\frac{x}{2}, \quad \mathcal{E}_{\log _{2}}\left(\rho_{N}(x)\right)=\log _{2} \frac{2}{2-x},
$$

and one of the optimal decompositions is

$$
\rho_{N}(x)=\frac{1}{4 N} \sum_{k=1}^{N} \sum_{\alpha= \pm} \sum_{\beta=u, v}\left|\psi_{\alpha, \beta, k}(x)\right\rangle\left\langle\psi_{\alpha, \beta, k}(x)\right| .
$$

The above calculations show that for $\rho_{N}(x)$, the entanglement depends on the portion $x$ of the GHZ state in states $\left|\psi_{\alpha, \beta, k}(x)\right\rangle\left\langle\psi_{\alpha, \beta, k}(x)\right|$ and it never becomes zero unless there is no GHZ mixture.

We conjecture that, for $N \geq 4, \rho_{N}(x)$ has $E_{R}(x)=x$, with one closest separable mixed state being

$$
\frac{x}{2}(|0 . .0\rangle\langle 0 . .0|+| 1 . .1\rangle\langle 1 . .1|)+\frac{1-x}{2 N} \sum_{k=1}^{N}\left(P_{k}+\bar{P}_{k}\right),
$$


which seems plausible as $(|0 . .0\rangle\langle 0 . .0|+| 1 . .1\rangle\langle 1 . .1|)$ is a closest separable mixed state to $\left|\Psi_{G}\right\rangle$.

Concluding remarks - We have presented analytical results on how much entanglement is bound in two distinct multipartite bound entangled states. The measure we have used to quantify their entanglement is the geometric measure of entanglement (GME), whose construction, similiar to the entanglement of formation $\left(E_{\mathrm{F}}\right)$, is via the convex hull. In contrast to GME, $E_{\mathrm{F}}$ has not been explicitly generalized to multipartite states, and hence is still unavailable for these bound entangled states. However, under the circumstances discussed previously, the results for $E_{\mathrm{sin}^{2}}$ as well as a related quantity, $\mathcal{E}_{\log _{2}}$, might provide lower bounds on $E_{\mathrm{F}}$. For the Smolin state, its bound entanglement is as large as that of a four-partite GHZ state, whereas that for Dür states is related to the portion of the $N$-partite GHZ state. For each case, an optimal decomposition is given. Furthermore, we have conjectured that the relative entropy of entanglement $\left(E_{\mathrm{R}}\right)$ for the Smolin state is unity (proved below), whereas we conjecture that $E_{\mathrm{R}}$ for Dür's state is equal to the portion that is $N$-partite GHZ.

For Smolin's state we can establish its $E_{\mathrm{F}}, E_{\mathrm{D}}, E_{\mathrm{R}}$, and $E_{\sin ^{2}}$ for certain bipartite partitionings. For example, if we group the four parties $\mathrm{ABCD}$ in two, $\mathrm{A}: \mathrm{BCD}$, we can write the state as

$$
\rho^{A: B C D}=\frac{1}{4} \sum_{i=0}^{3}\left|\bar{X}_{i}\right\rangle\left\langle\bar{X}_{i}\right|,
$$

with the 3-qubit states of BCD mapped on to the 8-level system $(000 \rightarrow \underline{0}, 001 \rightarrow \underline{1}, \ldots, 111 \rightarrow \underline{7})$, involving the locally orthogonal and convertible states (by BCD)

$$
\begin{array}{ll}
\left|\bar{X}_{0}\right\rangle=(|0 \underline{0}\rangle+|1 \underline{7}\rangle) / \sqrt{2}, & \left|\bar{X}_{1}\right\rangle=(|0 \underline{3}\rangle+|1 \underline{4}\rangle) / \sqrt{2}, \\
\left|\bar{X}_{2}\right\rangle=(|0 \underline{5}\rangle+|1 \underline{2}\rangle) / \sqrt{2}, & \left|\bar{X}_{3}\right\rangle=(|0 \underline{6}\rangle+|1 \underline{1}\rangle) / \sqrt{2} .
\end{array}
$$

In order to find the entanglement of this bipartite state (in $C^{2} \otimes C^{8}$ ), we need to consider the entanglement of the general (properly normalized) pure state

$$
|\psi\rangle \equiv \sum_{i} \sqrt{x_{i}} e^{i \phi_{i}}\left|\bar{X}_{i}\right\rangle
$$

that appears in the pure-state decompositions. In fact, regardless of the values of the $x_{i}$ 's, this pure state has a reduced density matrix (tracing over $\mathrm{BCD}$ ) of the form $(|0\rangle\langle 0|+| 1\rangle\langle 1|) / 2$. This shows that $\rho^{A: B C D}$ has $E_{\mathrm{F}}=1$, $E_{\sin ^{2}}=1 / 2$, and $\mathcal{E}_{\log _{2}}=1$. In fact, there is a general result due to Horodecki et al. [16] that $E_{\mathrm{D}}=E_{\mathrm{F}}$ for mixtures of locally orthogonal bipartite states, e.g., $C^{2} \otimes$ $C^{2 m}$ states that are derived from mixing Bell-like states

$$
\left|\Psi_{k}^{ \pm}\right\rangle \equiv(|0, \underline{k}\rangle \pm|1, \underline{2 m-k-1}\rangle) / \sqrt{2},
$$

having distinct $k$ 's, where $k=0,1, \ldots, m-1$. As $E_{\mathrm{D}} \leq E_{\mathrm{R}} \leq E_{\mathrm{F}}$, we have that $E_{\mathrm{R}}\left(\rho^{\mathrm{A}: \mathrm{BCD}}\right)=1$ as well.
What about the original four-partite state $\rho^{\mathrm{ABCD}}$ ? As $E_{\mathrm{R}}\left(\rho^{\mathrm{ABCD}}\right) \geq E_{\mathrm{R}}\left(\rho^{\mathrm{A}: \mathrm{BCD}}\right)$, we have $E_{\mathrm{R}}\left(\rho^{\mathrm{ABCD}}\right) \geq 1$. But we also have that $E_{\mathrm{R}}\left(\rho^{\mathrm{ABCD}}\right) \leq 1$, as our previous conjecture gives at least an upper bound; we thus have that $E_{\mathrm{R}}\left(\rho^{\mathrm{ABCD}}\right)=1$ and the conjecture is proved. Naively, we expect that any arbitrary $\rho^{\mathrm{ABCD}}$ has greater entanglement than $\rho^{\mathrm{A}: \mathrm{BCD}}$. However, for the Smolin state, they have the same entanglement as quantified by both GME and the relative entropy of entanglement.

Although Dür's bound entangled state violates a Bell inequality, it has nonzero negativity under certain partitionings. One may raise the question: Does there exist a bound entangled state that has positive PT (PPT) under all partitionings but that still violates a Bell's inequality? For example, does an unextendible-product-basis (UPB) bound entangled state [17] violate a Bell inequality? We shall see shortly that the answer is "no", at least for the three different Bell inequalities [10, 13, 14] mentioned earlier. Acín has shown [18] that if an $N$-qubit state violates a two-setting Bell inequality then it is distillable under certain bipartite partitioning. Using the results of Refs. 19, 20] regarding distillability, we can repeat the same analysis for the other two inequalities 13, 14] and indeed obtain the same conclusion; see App. B. This bipartite distillability then implies a negative PT (NPT) under that bipartite partitioning according to Horodecki et al. 3] Hence, violating these Bell inequalities implies NPT under certain bipartite partitioning. Said equivalently, if an $N$-qubit state has PPT under all bipartite partitionings then the state never violates these Bell inequalities. This seems to suggest that PPT bound entangled states are truly bound in nature that cannot give deviation from local theories.

Acknowledgments: We thank M. Ericsson, P. Kwiat, S. Mukhopadhyay, O. Rudolph and especially W. Dür for discussions. This work was supported by NSF Grant No. EIA01-21568 and DOE Grant No. DEFG02-91ER45439.

Appendix A: In this appendix we sketch proofs of two useful inequalities and describe the deriviation of the entanglement eigenvalue for the states in Eqs. (14). We start with the first sought inequality:

$$
\begin{aligned}
& \left(c_{1} c_{2} c_{3} c_{4}+s_{1} s_{2} s_{3} s_{4}\right)^{2}+\left(c_{1} c_{2} s_{3} s_{4}+s_{1} s_{2} c_{3} c_{4}\right)^{2} \\
& +\left(c_{1} s_{2} c_{3} s_{4}+s_{1} c_{2} s_{3} s_{4}\right)^{2}+\left(c_{1} s_{2} s_{3} c_{4}+s_{1} c_{2} c_{3} s_{4}\right)^{2} \leq 1 .
\end{aligned}
$$

We have simplified the notation by using $c_{i} \equiv \cos \theta_{i}$ and $s_{i} \equiv \sin \theta_{i}$. By subtracting the left-hand side from 1 and doing some algebraic manipulation, we arrive at the non-negative expression (hence the sought result):

$$
\begin{aligned}
& \left(c_{1} c_{2} c_{3} s_{4}-s_{1} s_{2} s_{3} c_{4}\right)^{2}+\left(c_{1} c_{2} s_{3} s_{4}-s_{1} s_{2} c_{3} s_{4}\right)^{2}+ \\
& \left(c_{1} s_{2} c_{3} c_{4}-s_{1} c_{2} s_{3} s_{4}\right)^{2}+\left(s_{1} c_{2} c_{3} c_{4}-c_{1} s_{2} s_{3} s_{4}\right)^{2} .
\end{aligned}
$$


The next sought inequality is (for $N \geq 4$ ):

$$
\begin{aligned}
& f_{N} \equiv\left(c_{1} \cdots c_{N}+s_{1} \cdots s_{N}\right)^{2}+ \\
& \quad \sum_{k=1}^{N}\left\{\left(c_{1} \cdots s_{k} \cdots c_{N}\right)^{2}+\left(s_{1} \cdots c_{k} \cdots s_{N}\right)^{2}\right\} \leq 1 .
\end{aligned}
$$

First, making similar arguments, one can show that $f_{4} \leq 1$. One can also show that $f_{N+1} \leq f_{N}$. Thus, by induction, we have the sought result.

We now discuss why $\sqrt{y}\left|\Psi_{G}\right\rangle \pm \sqrt{1-y}\left|u_{k}\right\rangle$ and $\sqrt{y}\left|\Psi_{G}\right\rangle \pm \sqrt{1-y}\left|v_{k}\right\rangle$ have as their maximal entanglement eigenvalue $\Lambda_{\max }(y)=\sqrt{(2-y) / 2}$. As one can make local relative phase shifts to transform $\sqrt{y}\left|\Psi_{G}\right\rangle+\sqrt{1-y}\left|u_{k}\right\rangle$ to $\sqrt{y}\left|\Psi_{G}\right\rangle-\sqrt{1-y}\left|u_{k}\right\rangle$, they have the same entanglement. The change from $\sqrt{y}\left|\Psi_{G}\right\rangle \pm \sqrt{1-y}\left|u_{k}\right\rangle$ to $\sqrt{y}\left|\Psi_{G}\right\rangle \pm \sqrt{1-y}\left|v_{k}\right\rangle$ is simply a flipping of 0 to 1 , and vice versa. The mapping from $k$ to $k^{\prime}$ is just a relabeling of parties. Thus, we need only consider the state

$$
\sqrt{y / 2}(|00 \cdots 0\rangle+|11 \cdots 1\rangle)+\sqrt{1-y}|10 \cdots 0\rangle .
$$

As this state is invariant under permutation of all parties except the first one, and as the coefficients are nonnegative, in order to find the maximal overlap we can make the hypothesis that the closest separable state is of the form

$$
(\sqrt{p}|0\rangle+\sqrt{1-p}|1\rangle) \otimes(\sqrt{q}|0\rangle+\sqrt{1-q}|1\rangle)^{\otimes N-1} .
$$

We further see that in order for the overlap to be maximal, $q$ must be either 1 or 0 . For the former case, we can further maximize the overlap to get $\sqrt{(2-y) / 2}$. For the latter case, the maximum overlap is $\sqrt{y / 2}$, which is less than $\sqrt{(2-y) / 2}$ (as $0 \leq y \leq 1)$. Hence, the state $\sqrt{y}\left|\Psi_{G}\right\rangle \pm \sqrt{1-y}\left|u_{k}\right\rangle$ has the entanglement eigenvalue $\sqrt{(2-y) / 2}$.

Appendix $B$ : In this appendix we analyze the connection between violation of three Bell inequalities and bipartite distillability as was done in Ref. 18 for the two-setting inequality. It was shown by Dür and Cirac 20] that an arbitrary $N$-qubit state $\rho$ can be locally depolarized into the form

$$
\begin{aligned}
\rho_{N} & =\lambda_{0}^{+}\left|\Psi_{0}^{+}\right\rangle\left\langle\Psi_{0}^{+}\left|+\lambda_{0}^{-}\right| \Psi_{0}^{-}\right\rangle\left\langle\Psi_{0}^{-}\right| \\
& +\sum_{j=1}^{2^{N-1}-1} \lambda_{j}\left(\left|\Psi_{j}^{+}\right\rangle\left\langle\Psi_{j}^{+}|+| \Psi_{j}^{-}\right\rangle\left\langle\Psi_{j}^{-}\right|\right),
\end{aligned}
$$

while preserving $\lambda_{0}^{ \pm}=\left\langle\Psi_{0}^{ \pm}|\rho| \Psi_{0}^{ \pm}\right\rangle$and $\lambda_{j}=\left\langle\Psi_{j}^{+}|\rho| \Psi_{j}^{+}\right\rangle+$ $\left\langle\Psi_{j}^{-}|\rho| \Psi_{j}^{-}\right\rangle$, where $\left|\Psi_{0}^{ \pm}\right\rangle \equiv\left(\left|0^{\otimes N}\right\rangle \pm\left|1^{\otimes N}\right\rangle\right) / \sqrt{2}$, and the $\left|\Psi_{j}^{ \pm}\right\rangle$'s are GHZ-like states, i.e., the states in Eq. (18), unfolded into qubit notation. Normalization gives the condition

$$
\lambda_{0}^{+}+\lambda_{0}^{-}+2 \sum_{j} \lambda_{j}=1
$$

Now define $\Delta \equiv \lambda_{0}^{+}-\lambda_{0}^{-}$, which we assume to be nonnegative without loss of generality. The condition that there is no bipartite distillability for some bipartite partitioning $P_{j}$ is [19]

$$
2 \lambda_{j} \geq \Delta
$$

Assuming nondistillability for all bipartite splittings, we have

$$
2 \sum_{j} \lambda_{j}=1-\left(\lambda_{0}^{+}+\lambda_{0}^{-}\right) \geq\left(2^{N-1}-1\right) \Delta .
$$

As $\lambda_{0}^{+}+\lambda_{0}^{-} \geq \Delta$, we have further that

$$
1-\Delta \geq\left(2^{N-1}-1\right) \Delta .
$$

For the two-setting Bell inequality considered by Acín [18], violation implies $\Delta>1 / 2^{(N-1) / 2}$. For the three-setting Bell inequality considered in [13], violation implies $\Delta>\sqrt{3}\left(2^{N} / 3^{N}\right)$. For the functional Bell inequality in [14], violation implies $\Delta>2\left(2^{N} / \pi^{N}\right)$. One can easily check that the three Bell inequalities considered are inconsistent with the non-bipartite-distillability condition, Eq. (19). Hence, the violating of these three Bell inequalities implies the existence of some bipartite distillability.

[1] M. Nielsen and I. Chuang, Quantum Computation and Quantum Information (Cambridge Univ. Press, 2000).

[2] For a review, see M. Horodecki, Quant. Info. Comp. 1, 3 (2001), and references therein.

[3] M. Horodecki, P. Horodecki, and R. Horodecki, Phys. Rev. Lett. 80, 5239 (1998).

[4] M. B. Plenio and V. Vedral, J. Phys. A 34, 6997 (2001).

[5] A. Shimony, Ann. NY. Acad. Sci. 755, 675 (1995).

[6] H. Barnum and N. Linden, J. Phys. A 34, 6787 (2001).

[7] T.-C. Wei and P. M. Goldbart, Phys. Rev. A 68, 042307 (2003).

[8] J. A. Smolin, Phys. Rev. A 63, 032306 (2001).

[9] P. W. Shor, J. A. Smolin, and A. V. Thapliyal, Phys. Rev. Lett. 90, 107901 (2003).

[10] W. Dür, Phys. Rev. Lett. 87, 230402 (2001).

[11] V. Vedral and M. B. Plenio, Phys. Rev. A 57, 1619 (1998).

[12] T.-C. Wei, M. Ericsson, P. M. Goldbart, and W. J. Munro, Quantum Inf. Comput. 4, 252 (2004); eprint quant-ph/0405002

[13] D. Kaszlikowski, L. C. Kwek, J. Chen, and C. h. Oh, Phys. Rev. A 66, 052309 (2002).

[14] A. Sen(De), U. Sen, and M. Żukowski, Phys. Rev. A 66, 062318 (2002).

[15] In arriving at this result we have made a hypothesis about the form of the closest separable pure state; see App. A. The result has been verified numerically for the $N=4$ case.

[16] P. Horodecki, M. Horodecki, and R. Horodecki, Acta Phys. Slov. 48, 144 (1998). 
[17] C. H. Bennett, D. P. DiVincenzo, T. Mor, P. W. Shor, J. A. Smolin, and B. M. Terhal, Phys. Rev. Lett. 82, 5385 (1999).

[18] A. Acín, Phys. Rev. Lett. 88, 027901 (2002).
[19] W. Dür and J. I. Cirac, Phys. Rev. A 62, 022302 (2000).

[20] W. Dür and J. I. Cirac, Phys. Rev. A 61, 042314 (2000). 